\title{
Interactive comment on "Seasonal
} statistical-dynamical prediction of the North Atlantic Oscillation by probabilistic post-processing" by André Düsterhus

\section{Anonymous Referee \#1}

Received and published: 21 October 2019

The introduction would benefit from a paragraph placing the proposed method in context with similar existing methods such as Bayesian Model Averaging, see Williams et al. (2014) https://doi.org/10.1002/qj.2198 for a review.

Interactive comment on Nonlin. Processes Geophys. Discuss., https://doi.org/10.5194/npg2019-50, 2019. 